\title{
Article \\ Uveitis and Other Ocular Complications Following COVID-19 Vaccination
}

\author{
Elena Bolletta ${ }^{1}(\mathbb{D})$, Danilo Iannetta ${ }^{2}$, Valentina Mastrofilippo ${ }^{1}$, Luca De Simone ${ }^{1}\left(\mathbb{D}\right.$, Fabrizio Gozzi ${ }^{1}$ (D), \\ Stefania Croci $^{3}{ }^{(D)}$, Martina Bonacini ${ }^{3}{ }^{(D}$, Lucia Belloni ${ }^{3}$, Alessandro Zerbini ${ }^{3}$, Chantal Adani ${ }^{1}$ (D), \\ Luigi Fontana ${ }^{4}\left(\mathbb{D}\right.$, Carlo Salvarani ${ }^{5,6}$ and Luca Cimino ${ }^{1,6, *(\mathbb{D})}$
}

1 Ocular Immunology Unit, Azienda USL-IRCCS, 42123 Reggio Emilia, Italy; elena.bolletta@ausl.re.it (E.B.); valentina.mastrofilippo@ausl.re.it (V.M.); luca.desimone@ausl.re.it (L.D.S.); fabrizio.gozzi@ausl.re.it (F.G.); chantal.adani@ausl.re.it (C.A.)

2 Ophthalmology Unit, Azienda USL-IRCCS, 42123 Reggio Emilia, Italy; danilo.iannetta@ausl.re.it

3 Clinical Immunology, Allergy and Advanced Biotechnologies Unit, Azienda USL-IRCCS, 42123 Reggio Emilia, Italy; stefania.croci@ausl.re.it (S.C.); martina.bonacini@ausl.re.it (M.B.); lucia.belloni@ausl.re.it (L.B.); alessandro.zerbini@ausl.re.it (A.Z.)

4 Ophthalmology Unit, DIMES, Alma Mater Studiorum, University of Bologna, S. Orsola-Malpighi Teaching Hospital, 40138 Bologna, Italy; luifonta@gmail.com

5 Rheumatology Unit, Azienda USL-IRCCS, 42123 Reggio Emilia, Italy; carlo.salvarani@ausl.re.it

6 Department of Surgery, Medicine, Dentistry and Morphological Sciences, with Interest in Transplants, Oncology and Regenerative Medicine, University of Modena and Reggio Emilia, 41124 Modena, Italy

* Correspondence: luca.cimino@ausl.re.it

check for updates

Citation: Bolletta, E.; Iannetta, D.; Mastrofilippo, V.; De Simone, L.; Gozzi, F.; Croci, S.; Bonacini, M.; Belloni, L.; Zerbini, A.; Adani, C.; et al. Uveitis and Other Ocular Complications Following COVID-19 Vaccination. J. Clin. Med. 2021, 10, 5960. https://doi.org/10.3390/ jcm10245960

Academic Editor: Ivo Guber

Received: 19 November 2021 Accepted: 17 December 2021 Published: 19 December 2021

Publisher's Note: MDPI stays neutral with regard to jurisdictional claims in published maps and institutional affiliations.

Copyright: (c) 2021 by the authors. Licensee MDPI, Basel, Switzerland. This article is an open access article distributed under the terms and conditions of the Creative Commons Attribution (CC BY) license (https:// creativecommons.org/licenses/by/ $4.0 /)$.

\begin{abstract}
Coronavirus disease 2019 (COVID-19) vaccines can cause transient local and systemic post-vaccination reactions. The aim of this study was to report uveitis and other ocular complications following COVID-19 vaccination. The study included 42 eyes of 34 patients (20 females, 14 males), with a mean age of 49.8 years (range 18-83 years). The cases reported were three herpetic keratitis, two anterior scleritis, five anterior uveitis (AU), three toxoplasma retinochoroiditis, two Vogt-KoyanagiHarada (VKH) disease reactivations, two pars planitis, two retinal vasculitis, one bilateral panuveitis in new-onset Behçet's disease, three multiple evanescent white dot syndromes (MEWDS), one acute macular neuroretinopathy (AMN), five retinal vein occlusions (RVO), one non-arteritic ischemic optic neuropathy (NAION), three activations of quiescent choroidal neovascularization (CNV) secondary to myopia or uveitis, and one central serous chorioretinopathy (CSCR). Mean time between vaccination and ocular complication onset was 9.4 days (range 1-30 days). Twenty-three cases occurred after Pfizer-BioNTech vaccination (BNT162b2 mRNA), 7 after Oxford-AstraZeneca vaccine (ChAdOx1 nCoV-19), 3 after ModernaTX vaccination (mRNA-1273), and 1 after Janssen Johnson \& Johnson vaccine (Ad26.COV2). Uveitis and other ocular complications may develop after the administration of COVID-19 vaccine.
\end{abstract}

Keywords: SARS-CoV-2 disease; COVID-19; vaccine; vaccination; uveitis; vaccine-associated uveitis; ocular complications

\section{Introduction}

Severe acute respiratory syndrome coronavirus 2 (SARS-CoV-2) causes the coronavirus disease 2019 (COVID-19), a multisystemic disorder with medical and socioeconomic consequences that have led to public health crises worldwide. In an effort to alleviate the morbidity and mortality associated with COVID-19 and arrest viral transmission, different types of vaccinations have been developed. Among these vaccines are the inactivated vaccines (PiCoVacc, Sinovac [1]; BBIBP-CorV, Sinopharm [2]), the viral vector vaccines (Ad26.COV2, Janssen Johnson \& Johnson [3]; ChAdOx1 nCoV-19/AZD1222, OxfordAstraZeneca [4]), the messenger ribonucleic acid (mRNA)-based vaccines (BNT162b2, 
Pfizer-BioNTech [5]; mRNA-1273, ModernaTX [6]), and the protein subunit vaccine (NVXCoV2373, Novavax [7]).

Common transient local and systemic post-vaccination reactions are pain, redness and/or swelling at the injection site, fatigue, headache, muscle pain, chills, fever, and nausea $[4,5]$. Although less common, other vaccine-related side effects include cutaneous reactions such as varicella zoster and herpes simplex flares [8].

Different types of ocular complications have also been reported after COVID-19 vaccination, including facial nerve palsy, abducens nerve palsy, new-onset Graves' disease, episcleritis, anterior scleritis, anterior uveitis (AU), multifocal choroiditis, reactivation of Vogt-Koyanagi-Harada (VKH) disease, multiple evanescent white dot syndrome (MEWDS), acute macular neuroretinopathy (AMN), paracentral acute middle maculopathy (PAAM), thrombosis, and central serous retinopathy (CSR) [9].

The aim of this study was to report uveitis and other cases of ocular complications following COVID-19 vaccination.

\section{Materials and Methods}

This retrospective study included patients with uveitis and other ocular complications following COVID-19 vaccination between January 2021 and October 2021 at the Ocular Immunology Unit, Azienda Unità Sanitaria Locale (AUSL)-IRCCS, Reggio Emilia, Italy.

Data collection consisted of demographic and clinical data. The demographic data included age, sex, general medical and ocular history, and medications. Clinical data included systemic and ocular symptoms post-vaccination, type of vaccine, time interval between vaccination (first and second dose) and symptom onset, laterality of eye disease, ocular findings, treatment, and outcome.

All patients underwent a complete ophthalmic examination with measurement of the best-corrected visual acuity (BCVA), anterior segment slit lamp biomicroscopy, fundus examination, and optical coherence tomography (OCT). Uveitis was graded and classified according to the Standardization of Uveitis Nomenclature (SUN) classification system [10]. In patients with a history of uveitis, the time interval from the last uveitis attack to current uveitis was calculated. In cases of de novo uveitis and no history of uveitis-related systemic disease, laboratory tests were performed at the discretion of the treating ophthalmologist with the aim of excluding other causes of ocular inflammation. These included complete blood count, blood chemistry, erythrocyte sedimentation rate (ESR), C-reactive protein (CRP), Venereal Disease Research Laboratory test (VDRL), Treponema pallidum haemagglutination (TPHA), interferon-gamma release assay (QuantiFERON@-TB Gold test), serum angiotensin-converting enzyme (ACE), serum lysozyme, high-resolution computerized tomography (HRCT) of the chest, and magnetic resonance imaging (MRI) of the brain. In all patients, follow-up was carried out for a minimum of 3 months.

The study and data collection were conducted in agreement with the principles of the Declaration of Helsinki and approved by the local ethics committee (protocol $n$. 2021/0111389 Comitato Etico Provinciale di Reggio Emilia, Italy). Informed written consent was obtained from all patients.

\section{Results}

\subsection{Demographics and Clinical Data}

The study included 42 eyes of 34 patients (20 females, 14 males), with a mean age of 49.8 years (range 18-83 years). Patients' demographic data, medical history, type of vaccine, and systemic symptoms post-vaccination are reported in Table 1. 
Table 1. Patients' demographics, medical history, type of vaccine, and systemic symptoms post vaccination.

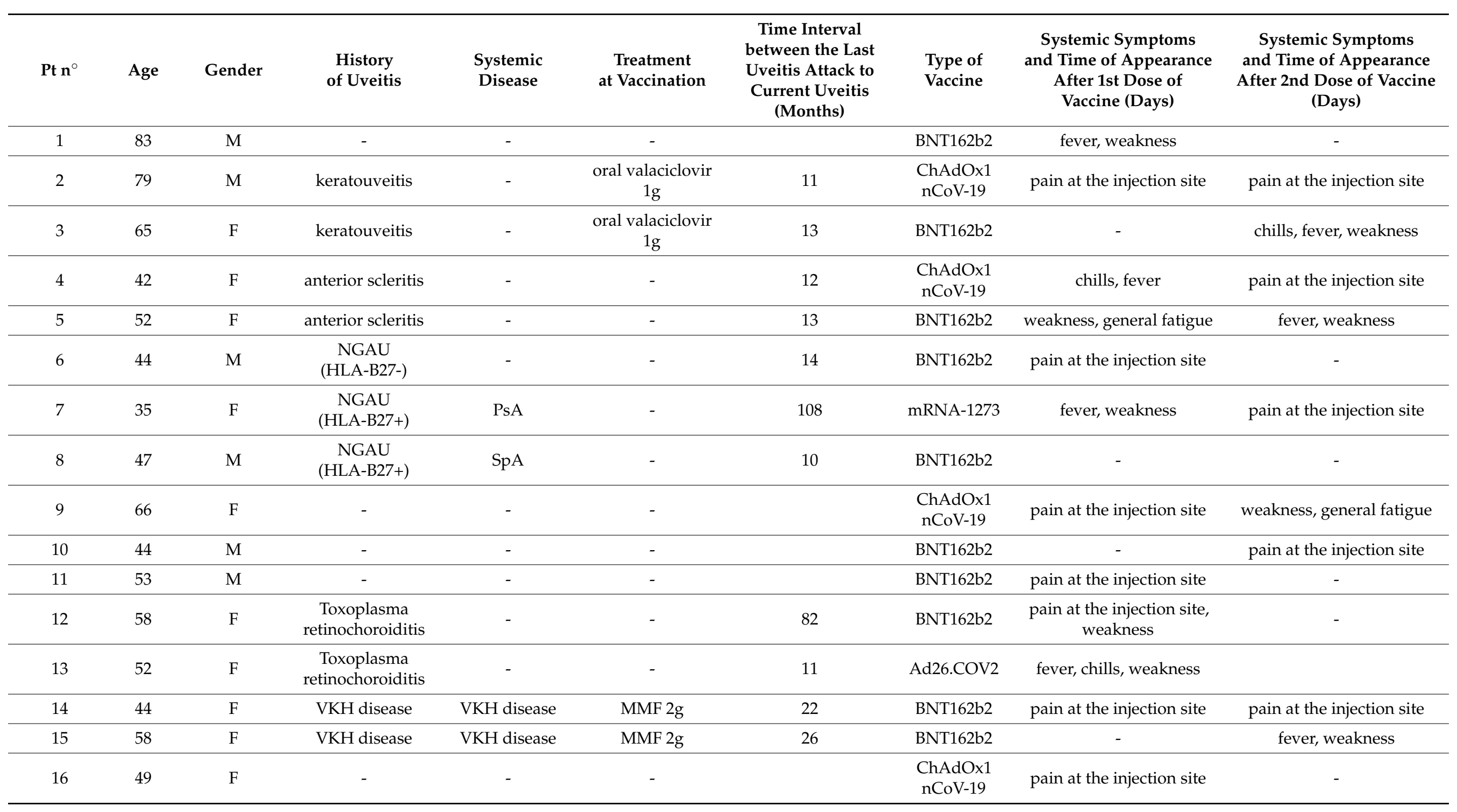


Table 1. Cont.

\begin{tabular}{|c|c|c|c|c|c|c|c|c|c|}
\hline $\operatorname{Pt}^{\circ}$ & Age & Gender & $\begin{array}{l}\text { History } \\
\text { of Uveitis }\end{array}$ & $\begin{array}{l}\text { Systemic } \\
\text { Disease }\end{array}$ & $\begin{array}{c}\text { Treatment } \\
\text { at Vaccination }\end{array}$ & $\begin{array}{c}\text { Time Interval } \\
\text { between the Last } \\
\text { Uveitis Attack to } \\
\text { Current Uveitis } \\
\text { (Months) }\end{array}$ & $\begin{array}{l}\text { Type of } \\
\text { Vaccine }\end{array}$ & $\begin{array}{c}\text { Systemic Symptoms } \\
\text { and Time of Appearance } \\
\text { After 1st Dose of } \\
\text { Vaccine (Days) }\end{array}$ & $\begin{array}{c}\text { Systemic Symptoms } \\
\text { and Time of Appearance } \\
\text { After 2nd Dose of Vaccine } \\
\text { (Days) }\end{array}$ \\
\hline 17 & 18 & $\mathrm{~F}$ & - & - & - & & BNT162b2 & - & fever \\
\hline 18 & 41 & $\mathrm{M}$ & - & - & - & & mRNA-1273 & fever, chills, weakness & pain at the injection site \\
\hline 19 & 59 & $\mathrm{~F}$ & - & - & - & & BNT162b2 & - & weakness \\
\hline 21 & 53 & $\mathrm{M}$ & - & - & - & & BNT162b2 & fever & - \\
\hline 22 & 18 & $\mathrm{~F}$ & - & - & - & & BNT162b2 & pain at the injection site & pain at the injection site \\
\hline 23 & 48 & $\mathrm{M}$ & - & - & - & & BNT162b2 & - & - \\
\hline 24 & 25 & $\mathrm{~F}$ & - & - & $\begin{array}{c}\text { estrogen- } \\
\text { progestin oral } \\
\text { contraceptives }\end{array}$ & & $\begin{array}{l}\text { ChAdOx1 } \\
\text { nCoV-19 }\end{array}$ & $\begin{array}{l}\text { pain at the injection site, } \\
\text { fever, muscle pain }\end{array}$ & \\
\hline 25 & 39 & $\mathrm{M}$ & - & - & - & & mRNA-1273 & fever, chills, muscle pain & pain at the injection site \\
\hline 26 & 53 & $\mathrm{~F}$ & - & SAH & $\begin{array}{c}\text { oral bisoprolol } \\
\text { and losartan }\end{array}$ & & $\begin{array}{l}\text { ChAdOx1 } \\
\text { nCoV-19 }\end{array}$ & $\begin{array}{l}\text { pain at the injection site, } \\
\text { weakness }\end{array}$ & - \\
\hline 27 & 61 & $\mathrm{~F}$ & - & - & - & & $\begin{array}{l}\text { ChAdOx1 } \\
\text { nCoV-19 }\end{array}$ & pain at the injection site & weakness \\
\hline 28 & 50 & $\mathrm{M}$ & - & $\mathrm{DM}$ & oral metformin & & BNT162b2 & - & weakness, fever \\
\hline 29 & 48 & $\mathrm{M}$ & - & SAH & oral doxazosin & & BNT162b2 & pain at the injection site & pain at the injection site \\
\hline 30 & 46 & $\mathrm{~F}$ & - & - & - & & BNT162b2 & pain at the injection site & - \\
\hline 31 & 47 & $\mathrm{~F}$ & $\begin{array}{l}\text { Toxoplasma } \\
\text { retinochoroiditis }\end{array}$ & - & - & 94 & BNT162b2 & $\begin{array}{l}\text { pain at the injection site, } \\
\text { chills, fever, weakness }\end{array}$ & - \\
\hline 32 & 68 & $\mathrm{~F}$ & $\begin{array}{l}\text { Serpiginous } \\
\text { Choroiditis }\end{array}$ & - & - & 13 & BNT162b2 & $\begin{array}{l}\text { pain at the injection site, } \\
\text { weakness }\end{array}$ & pain at the injection site \\
\hline 33 & 66 & $\mathrm{~F}$ & - & - & - & & BNT162b2 & - & pain at the injection site \\
\hline 34 & 41 & $\mathrm{M}$ & - & - & - & & BNT162b2 & pain at the injection site & weakness, muscle pain \\
\hline
\end{tabular}

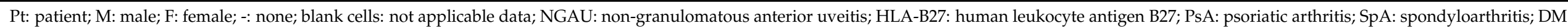
diabetes mellitus; SAH: systemic arterial hypertension; MMF: mycophenolate mofetil. 


\subsection{Uveitis and Other Ocular Complications}

Mean time between vaccination and ocular complications onset was 9.4 days (median time 7 days, range 1-30 days). Ocular complications reported after the first dose of the vaccine occurred at a mean time of 7.5 days (median time 6.5 days, range 2-30 days), while ocular complications after the second dose of vaccine were reported at a mean time of 10.7 days (median time 7 days, range 1-30 days). Eleven patients had a known history of uveitis and 2 of scleritis, the median time from previous to current attack was 13 months (range 10-108 months). Four patients had a uveitis-related systemic disease: one patient had psoriatic arthritis (PsA), one patient had spondyloarthritis (SpA), and two patients had VKH disease.

The study included three cases of herpetic keratitis, two anterior scleritis, five AU, three toxoplasma retinochoroiditis, two cases of VKH disease reactivation, two pars planitis, two retinal vasculitis, one bilateral panuveitis, three MEWDS, one AMN, five retinal vein occlusion (RVO), one non-arteritic ischemic optic neuropathy (NAION), three activations of quiescent choroidal neovascularization (CNV), and an acute-onset bilateral CSR (Table 2).

Among the three cases of herpetic keratitis, one patient had a previous history of herpetic keratitis and had not been taking systemic antiviral treatment before vaccination. The other two patients that reported herpetic keratitis had a previous history of herpetic keratouveitis and had $1 \mathrm{~g}$ of oral valacyclovir once daily for prophylactic therapy during vaccination. The mean time between vaccination and herpetic keratitis onset was 6 days (range 5-7 days).

The five cases of AU included one patient with CMV AU and four with non-granulomatous anterior uveitis (NGAU). Among NGAU were three patients with human leukocyte antigen B27 (HLA-B27) + and three with a previous history of uveitis, two of whom with uveitisrelated systemic disease (PsA and $\mathrm{SpA}$ ) had not been taking systemic therapy at vaccination.

Three patients reported ocular toxoplasmosis, at a mean time of 7.3 days (range 7-8 days) after the vaccination, one patient had an initial episode, and two patients had a recurrence of Toxoplasma retinochoroiditis.

Two patients with bilateral intermediate uveitis had negative laboratory tests and normal chest HRTC and MRI of the brain. Pars planitis was diagnosed in both patients.

One patient presented bilateral panuveitis with retinal vasculitis, papillary oedema, and painful oral ulcers, followed by deep vein thrombosis of a lower extremity. Laboratory workup showed elevated CRP, elevated ESR, and positive pathergy test with a diagnosis of Behçet's disease (BD).

The female patient who developed AMN 2 days after ChAdOx $1 \mathrm{nCoV}-19$ vaccine was taking combined estrogen-progestin oral contraceptives at the time of vaccination, which were immediately suspended.

Five patients developed RVO: one central retinal vein occlusion (CRVO) and four branch retinal vein occlusion (BRVO). Of these patients, one was affected by diabetes mellitus (DM) and two by systemic arterial hypertension (SAH) (Table 1).

A female patient of 46 years old presented unilateral sectorial papillary oedema, no crowded disc was observed in the other eye. Automatic perimetry demonstrated a diffuse visual field loss. The patient had no clinical signs of giant cell arteritis (jaw claudication, headache, and scalp tenderness), and CRP and ESR were within the normal range. Brain imaging excluded an acute intracranial event, and the patient was diagnosed with NAION. Systemic risk factors associated with NAION such as SAH, DM, hyperlipidemia, and smoking were negative. A hypercoagulable state due to antiphospholipid antibodies was ruled out. Also, anticardiolipin and lupus anticoagulant were negative.

Three cases presented activation of quiescent $\mathrm{CNV}$, secondary to myopia in one patient and secondary to uveitis in the other two. The myopic CNV and one uveitic CNV were never injected, while the other uveitic patient had the reactivation of a lesion treated with intraocular injections of anti-vascular endothelial growth factor (anti-VEGF) drugs 1 year earlier. 
Table 2. Uveitis and other ocular complications post vaccination.

\begin{tabular}{|c|c|c|c|c|c|c|c|c|c|c|}
\hline $\operatorname{Pt~n}^{\circ}$ & Eye & $\begin{array}{c}\text { Ocular } \\
\text { Complication }\end{array}$ & $\begin{array}{l}\text { History } \\
\text { of Uveitis }\end{array}$ & $\begin{array}{c}\text { Ocular } \\
\text { Complication } \\
\text { Following 1st or } \\
\text { 2nd Dose of } \\
\text { Vaccine }\end{array}$ & $\begin{array}{c}\text { Time Interval from } \\
\text { Vaccine to Ocular } \\
\text { Symptoms Onset } \\
\text { (Days) }\end{array}$ & $\begin{array}{l}\text { Ocular } \\
\text { Symptoms }\end{array}$ & $\begin{array}{c}\text { BCVA at } \\
\text { Presentation } \\
\text { (Snellen) }\end{array}$ & $\begin{array}{l}\text { BCVA at Last } \\
\text { Follow Up } \\
\text { (Snellen) }\end{array}$ & $\begin{array}{l}\text { Treatment Given } \\
\text { at Presentation }\end{array}$ & Outcome \\
\hline 1 & $\mathrm{LE}$ & herpetic keratitis & - & 2nd dose & 7 & $\begin{array}{l}\text { redness, pain, } \\
\text { blurred vision }\end{array}$ & $20 / 40$ & $20 / 25$ & $\begin{array}{l}\text { acyclovir } \\
\text { ophthalmic } \\
\text { ointment }\end{array}$ & $\begin{array}{l}\text { complete } \\
\text { resolution }\end{array}$ \\
\hline 2 & $\mathrm{RE}$ & herpetic keratitis & keratouveitis & 1st dose & 5 & $\begin{array}{c}\text { pain, } \\
\text { blurred vision }\end{array}$ & $20 / 40$ & $20 / 22$ & $\begin{array}{l}\text { oral valaciclovir } \\
1 \mathrm{~g}, \\
\text { dexamethasone } \\
\text { eye drops } 2 \\
\mathrm{mg} / \mathrm{ml}\end{array}$ & $\begin{array}{l}\text { complete } \\
\text { resolution }\end{array}$ \\
\hline 3 & LE & herpetic keratitis & keratouveitis & 2nd dose & 6 & $\begin{array}{c}\text { redness, } \\
\text { blurred vision }\end{array}$ & $20 / 50$ & $20 / 20$ & $\begin{array}{l}\text { oral valaciclovir } \\
1 \mathrm{~g}, \\
\text { dexamethasone } \\
\text { eye drops } 2 \\
\mathrm{mg} / \mathrm{ml}\end{array}$ & $\begin{array}{l}\text { complete } \\
\text { resolution }\end{array}$ \\
\hline 5 & $\mathrm{RE}$ & anterior scleritis & $\begin{array}{l}\text { anterior } \\
\text { scleritis }\end{array}$ & 1st dose & 5 & redness, pain & $20 / 20$ & $20 / 20$ & $\begin{array}{l}\text { dexamethasone } \\
\text { eye drops } 2 \\
\mathrm{mg} / \mathrm{ml}\end{array}$ & $\begin{array}{l}\text { complete } \\
\text { resolution }\end{array}$ \\
\hline 6 & $\mathrm{LE}$ & $\begin{array}{c}\text { NGAU } \\
\text { (HLA-B27-) }\end{array}$ & $\begin{array}{c}\text { NGAU } \\
\text { (HLA-B27-) }\end{array}$ & 1st dose & 6 & photophobia & $20 / 20$ & $20 / 20$ & $\begin{array}{l}\text { dexamethasone } \\
\text { eye drops } 2 \\
\mathrm{mg} / \mathrm{ml}\end{array}$ & $\begin{array}{l}\text { complete } \\
\text { resolution }\end{array}$ \\
\hline 7 & $\mathrm{BE}$ & $\begin{array}{c}\text { NGAU } \\
(\text { HLA-B27+) }\end{array}$ & $\begin{array}{c}\text { NGAU } \\
(\text { HLA-B27+) }\end{array}$ & 2nd dose & 1 & $\begin{array}{l}\text { redness, pain, } \\
\text { blurred vision }\end{array}$ & $\begin{array}{l}\text { RE: } 20 / 22 \\
\text { LE: } 20 / 25\end{array}$ & $\begin{array}{l}\text { RE: } 20 / 20 \\
\text { LE: } 20 / 22\end{array}$ & $\begin{array}{l}\text { dexamethasone } \\
\text { eye drops } 2 \\
\mathrm{mg} / \mathrm{ml}\end{array}$ & $\begin{array}{l}\text { complete } \\
\text { resolution }\end{array}$ \\
\hline 8 & LE & $\begin{array}{c}\text { NGAU } \\
(\text { HLA-B27+) }\end{array}$ & $\begin{array}{c}\text { NGAU } \\
(\text { HLA-B27+) }\end{array}$ & 2nd dose & 6 & blurred vision & $20 / 25$ & $20 / 20$ & $\begin{array}{l}\text { dexamethasone } \\
\text { eye drops } 2 \\
\mathrm{mg} / \mathrm{ml}\end{array}$ & $\begin{array}{l}\text { complete } \\
\text { resolution }\end{array}$ \\
\hline
\end{tabular}


Table 2. Cont.

\begin{tabular}{|c|c|c|c|c|c|c|c|c|c|c|}
\hline $\operatorname{Pt}^{\circ}$ & Eye & $\begin{array}{c}\text { Ocular } \\
\text { Complication }\end{array}$ & $\begin{array}{l}\text { History } \\
\text { of Uveitis }\end{array}$ & $\begin{array}{c}\text { Ocular } \\
\text { Complication } \\
\text { Following 1st or } \\
\text { 2nd Dose of } \\
\text { Vaccine }\end{array}$ & $\begin{array}{c}\text { Time Interval from } \\
\text { Vaccine to Ocular } \\
\text { Symptoms Onset } \\
\text { (Days) }\end{array}$ & $\begin{array}{l}\text { Ocular } \\
\text { Symptoms }\end{array}$ & $\begin{array}{l}\text { BCVA at } \\
\text { Presentation } \\
\text { (Snellen) }\end{array}$ & $\begin{array}{l}\text { BCVA at Last } \\
\text { Follow Up } \\
\text { (Snellen) }\end{array}$ & $\begin{array}{l}\text { Treatment Given } \\
\text { at Presentation }\end{array}$ & Outcome \\
\hline 9 & LE & $\begin{array}{c}\text { NGAU } \\
(\text { HLA-B27+) }\end{array}$ & - & 1st dose & 30 & $\begin{array}{l}\text { redness, pain, } \\
\text { blurred vision }\end{array}$ & $20 / 32$ & $20 / 25$ & $\begin{array}{l}\text { dexamethasone } \\
\text { eye drops } 2 \\
\mathrm{mg} / \mathrm{ml}\end{array}$ & $\begin{array}{l}\text { complete } \\
\text { resolution }\end{array}$ \\
\hline 10 & $\mathrm{RE}$ & CMV AU & - & 2nd dose & 8 & blurred vision & $20 / 28$ & $20 / 20$ & $\begin{array}{c}\text { ganciclovir } \\
\text { ophthalmic gel } \\
0.15 \%, \\
\text { dexamethasone } \\
\text { eye drops } 2 \\
\mathrm{mg} / \mathrm{ml}\end{array}$ & $\begin{array}{l}\text { complete } \\
\text { resolution }\end{array}$ \\
\hline 11 & LE & $\begin{array}{c}\text { Toxoplasma } \\
\text { retinochoroiditis }\end{array}$ & - & 1st dose & 8 & blurred vision & $20 / 40$ & $20 / 20$ & $\begin{array}{l}\text { sulfadiazine and } \\
\text { pyrimethamine } \\
\text { tablets, oral } \\
\text { prednisone }\end{array}$ & $\begin{array}{l}\text { complete } \\
\text { resolution }\end{array}$ \\
\hline 13 & $\mathrm{RE}$ & $\begin{array}{l}\text { Toxoplasma } \\
\text { retinochoroiditis }\end{array}$ & $\begin{array}{l}\text { Toxoplasma } \\
\text { retinochoroiditis }\end{array}$ & 1st dose & 7 & blurred vision & $20 / 50$ & $20 / 20$ & $\begin{array}{l}\text { sulfadiazine and } \\
\text { pyrimethamine } \\
\text { tablets, oral } \\
\text { prednisone }\end{array}$ & $\begin{array}{l}\text { complete } \\
\text { resolution }\end{array}$ \\
\hline 14 & $\mathrm{BE}$ & VKH disease & VKH disease & 2nd dose & 12 & blurred vision & $\begin{array}{l}\text { RE: } 20 / 22 \\
\text { LE: } 20 / 25\end{array}$ & $\begin{array}{l}\text { RE: } 20 / 20 \\
\text { LE: } 20 / 20\end{array}$ & $\begin{array}{l}\text { MMF } 2 \mathrm{~g} \text {, oral } \\
\text { prednisone }\end{array}$ & $\begin{array}{l}\text { complete } \\
\text { resolution }\end{array}$ \\
\hline 15 & $\mathrm{BE}$ & VKH disease & VKH disease & 2nd dose & 5 & blurred vision & $\begin{array}{l}\text { RE: } 20 / 25 \\
\text { LE: } 20 / 28\end{array}$ & $\begin{array}{l}\text { RE: } 20 / 20 \\
\text { LE: } 20 / 22\end{array}$ & $\begin{array}{l}\text { MMF 2g, oral } \\
\text { prednisone }\end{array}$ & $\begin{array}{l}\text { complete } \\
\text { resolution }\end{array}$ \\
\hline 16 & $\mathrm{BE}$ & pars planitis & - & 1st dose & 7 & blurred vision & $\begin{array}{l}\text { RE: } 20 / 25 \\
\text { LE: } 20 / 20\end{array}$ & $\begin{array}{l}\text { RE: } 20 / 20 \\
\text { LE: } 20 / 20\end{array}$ & oral prednisone & $\begin{array}{l}\text { complete } \\
\text { resolution }\end{array}$ \\
\hline
\end{tabular}


Table 2. Cont.

\begin{tabular}{|c|c|c|c|c|c|c|c|c|c|c|}
\hline $\operatorname{Pt} n^{\circ}$ & Eye & $\begin{array}{c}\text { Ocular } \\
\text { Complication }\end{array}$ & $\begin{array}{l}\text { History } \\
\text { of Uveitis }\end{array}$ & $\begin{array}{c}\text { Ocular } \\
\text { Complication } \\
\text { Following 1st or } \\
\text { 2nd Dose of } \\
\text { Vaccine }\end{array}$ & $\begin{array}{l}\text { Time Interval from } \\
\text { Vaccine to Ocular } \\
\text { Symptoms Onset } \\
\text { (Days) }\end{array}$ & $\begin{array}{c}\text { Ocular } \\
\text { Symptoms }\end{array}$ & $\begin{array}{c}\text { BCVA at } \\
\text { Presentation } \\
\text { (Snellen) }\end{array}$ & $\begin{array}{l}\text { BCVA at Last } \\
\text { Follow Up } \\
\text { (Snellen) }\end{array}$ & $\begin{array}{l}\text { Treatment Given } \\
\text { at Presentation }\end{array}$ & Outcome \\
\hline 17 & $\mathrm{BE}$ & pars planitis & - & 2nd dose & 14 & blurred vision & $\begin{array}{l}\text { RE: } 20 / 20 \\
\text { LE: } 20 / 20\end{array}$ & $\begin{array}{l}\text { RE: } 20 / 20 \\
\text { LE: } 20 / 20\end{array}$ & oral prednisone & $\begin{array}{l}\text { complete } \\
\text { resolution }\end{array}$ \\
\hline 18 & $\mathrm{BE}$ & retinal vasculitis & - & 2nd dose & 5 & blurred vision & $\begin{array}{l}\text { RE: } 20 / 66 \\
\text { LE: } 20 / 20\end{array}$ & $\begin{array}{l}\text { RE: } 20 / 20 \\
\text { LE: } 20 / 20\end{array}$ & oral prednisone & $\begin{array}{l}\text { complete } \\
\text { resolution }\end{array}$ \\
\hline 19 & $\mathrm{RE}$ & retinal vasculitis & - & 1st dose & 10 & blurred vision & $20 / 28$ & $20 / 20$ & oral prednisone & $\begin{array}{l}\text { complete } \\
\text { resolution }\end{array}$ \\
\hline 20 & $\mathrm{BE}$ & $\begin{array}{l}\text { panuveitis in } \\
\text { new-onset BD }\end{array}$ & - & 2nd dose & 30 & $\begin{array}{c}\text { redness, } \\
\text { blurred vision }\end{array}$ & $\begin{array}{l}\text { RE: } 20 / 28 \\
\text { LE: } 20 / 32\end{array}$ & $\begin{array}{l}\text { RE: } 20 / 25 \\
\text { LE: } 20 / 22\end{array}$ & $\begin{array}{c}\text { oral prednisone, } \\
\text { AZA }\end{array}$ & $\begin{array}{l}\text { complete } \\
\text { resolution }\end{array}$ \\
\hline 21 & LE & MEWDS & - & 2nd dose & 28 & $\begin{array}{c}\text { decreased VA, } \\
\text { visual field } \\
\text { defect }\end{array}$ & $20 / 25$ & $20 / 20$ & - & $\begin{array}{l}\text { complete } \\
\text { resolution }\end{array}$ \\
\hline 22 & $\mathrm{RE}$ & MEWDS & - & 1st dose & 4 & $\begin{array}{l}\text { blurred vision, } \\
\text { visual field } \\
\text { defect }\end{array}$ & $20 / 66$ & $20 / 20$ & - & $\begin{array}{l}\text { complete } \\
\text { resolution }\end{array}$ \\
\hline 23 & $\mathrm{RE}$ & MEWDS & - & 1st dose & 7 & decreased VA & $20 / 400$ & $20 / 20$ & - & $\begin{array}{l}\text { complete } \\
\text { resolution }\end{array}$ \\
\hline 24 & $\mathrm{BE}$ & AMN & - & 1st dose & 2 & $\begin{array}{l}\text { visual field } \\
\text { defect }\end{array}$ & $\begin{array}{l}\text { RE: } 20 / 20 \\
\text { LE: } 20 / 20\end{array}$ & $\begin{array}{l}\text { RE: } 20 / 20 \\
\text { LE: } 20 / 20\end{array}$ & - & $\begin{array}{c}\text { significant } \\
\text { improvement }\end{array}$ \\
\hline 25 & $\mathrm{RE}$ & CRVO & - & 2nd dose & 30 & decreased VA & $20 / 400$ & $20 / 100$ & $\begin{array}{l}\text { intravitreal } \\
\text { anti-VEGF }\end{array}$ & $\begin{array}{c}\text { mild } \\
\text { improvement }\end{array}$ \\
\hline 26 & LE & BRVO & - & 1st dose & 2 & decreased VA & $20 / 100$ & $20 / 40$ & $\begin{array}{l}\text { intravitreal } \\
\text { anti-VEGF }\end{array}$ & $\begin{array}{c}\text { partial } \\
\text { improvement }\end{array}$ \\
\hline 27 & LE & BRVO & - & 2nd dose & 2 & decreased VA & $20 / 32$ & $20 / 25$ & $\begin{array}{l}\text { intravitreal } \\
\text { anti-VEGF }\end{array}$ & $\begin{array}{c}\text { partial } \\
\text { improvement }\end{array}$ \\
\hline 28 & LE & BRVO & - & 2nd dose & 3 & decreased VA & $20 / 22$ & $20 / 20$ & $\begin{array}{c}\text { intravitreal } \\
\text { anti-VEGF }\end{array}$ & $\begin{array}{c}\text { significant } \\
\text { improvement }\end{array}$ \\
\hline
\end{tabular}


Table 2. Cont.

\begin{tabular}{|c|c|c|c|c|c|c|c|c|c|c|}
\hline $\operatorname{Pt} \mathbf{n}^{\circ}$ & Eye & $\begin{array}{c}\text { Ocular } \\
\text { Complication }\end{array}$ & $\begin{array}{l}\text { History } \\
\text { of Uveitis }\end{array}$ & $\begin{array}{c}\text { Ocular } \\
\text { Complication } \\
\text { Following 1st or } \\
\text { 2nd Dose of } \\
\text { Vaccine }\end{array}$ & $\begin{array}{l}\text { Time Interval from } \\
\text { Vaccine to Ocular } \\
\text { Symptoms Onset } \\
\text { (Days) }\end{array}$ & $\begin{array}{c}\text { Ocular } \\
\text { Symptoms }\end{array}$ & $\begin{array}{l}\text { BCVA at } \\
\text { Presentation } \\
\text { (Snellen) }\end{array}$ & $\begin{array}{l}\text { BCVA at Last } \\
\text { Follow Up } \\
\text { (Snellen) }\end{array}$ & $\begin{array}{l}\text { Treatment Given } \\
\text { at Presentation }\end{array}$ & Outcome \\
\hline 29 & LE & BRVO & - & 2nd dose & 23 & blurred vision & $20 / 20$ & $20 / 20$ & $\begin{array}{l}\text { intravitreal } \\
\text { anti-VEGF }\end{array}$ & $\begin{array}{l}\text { significant } \\
\text { improvement }\end{array}$ \\
\hline 30 & $\mathrm{RE}$ & NAION & - & 1st dose & 2 & $\begin{array}{l}\text { decreased VA, } \\
\text { visual field } \\
\text { defect }\end{array}$ & $20 / 40$ & $20 / 200$ & oral prednisone & $\begin{array}{c}\text { no } \\
\text { improvement }\end{array}$ \\
\hline 31 & $\mathrm{RE}$ & uveitic CNV & $\begin{array}{l}\text { Toxoplasma } \\
\text { retinochoroiditis }\end{array}$ & 1st dose & 8 & decreased VA & $20 / 200$ & $20 / 40$ & $\begin{array}{l}\text { intravitreal } \\
\text { anti-VEGF }\end{array}$ & $\begin{array}{l}\text { significant } \\
\text { improvement }\end{array}$ \\
\hline 32 & $\mathrm{RE}$ & uveitic CNV & $\begin{array}{l}\text { Serpiginous } \\
\text { Choroiditis }\end{array}$ & 2nd dose & 10 & decreased VA & $20 / 50$ & $20 / 32$ & $\begin{array}{l}\text { intravitreal } \\
\text { anti-VEGF }\end{array}$ & $\begin{array}{c}\text { partial } \\
\text { improvement }\end{array}$ \\
\hline 33 & $\mathrm{RE}$ & myopic CNV & - & 2nd dose & 1 & blurred vision & $20 / 32$ & $20 / 25$ & $\begin{array}{l}\text { intravitreal } \\
\text { anti-VEGF }\end{array}$ & $\begin{array}{c}\text { partial } \\
\text { improvement }\end{array}$ \\
\hline 34 & $\mathrm{BE}$ & CSR & - & 2nd dose & 13 & blurred vision & $\begin{array}{l}\text { RE: } 20 / 22 \\
\text { LE: } 20 / 50\end{array}$ & $\begin{array}{l}\text { RE: } 20 / 20 \\
\text { LE: } 20 / 22\end{array}$ & - & $\begin{array}{l}\text { complete } \\
\text { resolution }\end{array}$ \\
\hline
\end{tabular}

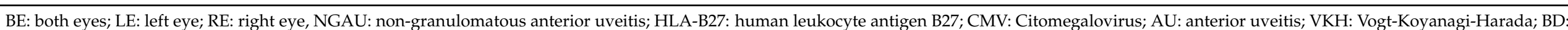

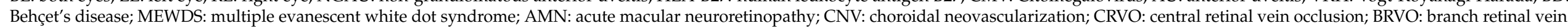

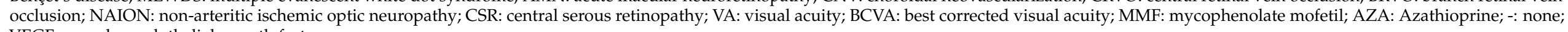
VEGF: vascular endothelial growth factor. 


\subsection{Type of Vaccine}

Twenty-three cases occurred after Pfizer-BioNTech vaccination (BNT162b2 mRNA), seven after Oxford-AstraZeneca vaccine (ChAdOx1 nCoV-19), three after ModernaTX vaccination (mRNA-1273), and one after Janssen Johnson \& Johnson vaccine (Ad26.COV2). Fourteen cases were reported after the first dose of the vaccine and 20 after the second dose (Table 2).

\section{Discussion}

Uveitis and other ocular adverse events have been described following vaccinations for hepatitis B virus (HBV), human papillomavirus (HPV), influenza virus, Bacille-CalmetteGuerin (BCG), varicella virus, measles-mumps-rubella (MMR), yellow fever, hepatitis A virus (HAV), and typhoid [11-15]. Different types of ocular complications have also been reported after COVID-19 vaccination. Pichi et al. reported one patient with episcleritis, two with anterior scleritis, two with AMN, one with PAAM, and one with subretinal fluid soon after receiving an inactivated COVID-19 vaccination (Sinopharm) [16].

A retrospective multicenter study collected 21 cases of unilateral or bilateral AU and 2 cases of MEWDS after the administration of the BNT162b2 mRNA vaccine [17]. Moreover, two case reports described bilateral multifocal choroiditis following COVID-19 vaccination $[18,19]$. Other cases of facial nerve palsy, abducens nerve palsy, new-onset Graves' disease, VKH disease reactivation, AMN, PAAM, and thrombosis have been described [9].

This retrospective study reports uveitis and other ocular complications following COVID-19 vaccination. We observed three cases of herpes keratitis reactivation following COVID-19 vaccination.

Vaccines can result in varicella zoster virus (VZV) reactivation, as previously described in patients receiving inactivated vaccines for hepatitis $\mathrm{A}$, influenza, rabies, and Japanese encephalitis. Fernandez-Nieto et al. described 15 cases of herpes simplex/zoster in patients infected with COVID-19 [20]. COVID-19 infection may represent a trigger for herpes reactivation, as recently reported. There are cases reported in the literature characterized by VZV reactivation after vaccination with the mRNA COVID-19 vaccine, including also herpes zoster ophthalmicus (HZO) [21-24]. It has been postulated that the stimulation of the immune system following vaccination induces a strong T-cell response with increased CD8+ T cell and T helper type 1 CD4+ T cells. Temporarily, VZV-specific CD8+ cells are not capable of controlling VZV after the massive shift of naïve CD8+ cells, which allows VZV to escape from its latent phase. Moreover, another possible explanation focuses on toll-like receptors (TLR) signaling. Abrogations in TLR expression among vaccinated individuals have been linked with marked induction of type I interferon (IFN-I) and potentiation of pro-inflammatory cytokines, which, although they promote $\mathrm{T}$ cell immunity and initiate an antibody-secreting memory $B$ cell response, may negatively modulate antigen expression while potentially contributing to VZV reactivation [25].

As reported in the literature, the median time between COVID-19 diagnosis and the development of herpes zoster was 5.5 days [26]. Similarly, the VZV reactivation appeared 5 days after COVID-19 vaccination in a case report [23]. In our three cases, herpes keratitis reactivation occurred after a mean of 6 days (range 5-7 days) after COVID-19 vaccination.

A recent case report of a severe unilateral flare-up of a granulomatous hypertensive uveitis 5 days after the second dose of Moderna vaccine in a patient previously treated for herpes keratouveitis suggests that preventive antiviral treatment should be given in known herpes patients despite quiescent uveitis to avoid potential reactivation [27]. In two of our cases, herpes keratitis reactivation happened in patients with a history of previous herpetic keratouveitis although under systemic antiviral treatment with oral valacyclovir of $1 \mathrm{~g}$ once daily.

Episcleritis has been described as ocular manifestations in patients with COVID19 [28-30]. Anterior scleritis has also been reported to manifest after COVID-19 [31]. In addition, scleritis and episcleritis have also been reported in three patients at a mean of 
5 days after the first dose of the inactivated COVID-19 vaccine (Sinopharm) [16]. Consistent with the reported literature, our two cases of scleritis were mild and noted at a mean of 5.5 days after vaccination.

In accordance with other studies, we reported AU in patients with or without a history of previous uveitis and/or uveitis-related systemic disease [17,32]. The vaccine-induced increase in IFN-I secretion could potentially drive autoimmune manifestations in patients with a history of autoimmunity or with yet unknown susceptibility to develop one [17,33].

Among our cases, two patients had a recurrence of Toxoplasma retinochoroiditis and one patient an initial episode of Toxoplasma retinochoroiditis at a mean of 7.3 days (range 7-8 days) after the vaccination. The vaccination-induced CD8 T-cell exhaustion may lead to parasite reactivation [34].

Papasavvas I. and Herbort CP. reported a case of VKH disease reactivation 6 weeks after the second dose of the Pfizer anti-SARS-CoV-2 vaccine administration that had been completely under control with a maintenance treatment of infliximab every 10 weeks for 6 years. The patient presented with a severe reactivation of the disease almost as pronounced as during its initial onset [35]. In our study, two patients with VKH disease being treated with mycophenolate mofetil ( $2 \mathrm{~g}$ daily) presented a mild reactivation with choroidal granulomas on indocyanine green angiography (ICGA).

Cases of COVID-19 associated with systemic vasculitis, including retinal vasculitis and papillophlebitis, have been published [36,37]. In our study, two patients presented with bilateral intermediate uveitis and two patients with bilateral retinal vasculitis. These four patients underwent blood tests, chest HRCT, and MRI of the brain, all of which were negative.

A single case of clinical presentation consistent with new-onset BD or a BD-like adverse event following SARS-CoV-2 mRNA-1273 vaccination has been described [38]. Similarly, we reported a case of bilateral panuveitis with new-onset BD.

A multicenter study reported two cases of MEWDS occurring 5 and 30 days after BNT162b2 mRNA vaccination [17]. Our three cases of MEWDS were reported from 4 to 28 days after the first or second dose of BNT162b2 mRNA vaccination.

PAMM and AMN have been reported after H1N1 vaccination. Virgo and Mohamed reported two patients with new paracentral scotoma secondary to AMN and PAMM 16 days after confirmed COVID-19 infections [39]. Furthermore, four case reports described five cases of AMN in young women 2 days after receiving ChAdOx1 nCoV-19 vaccination [40-43]. Similarly, a case of AMN occurred in a young woman in our study that was taking combined estrogen-progestin oral contraceptives 2 days after ChAdOx1 nCoV-19 vaccination $[40,42,43]$.

Artery or vein retinal occlusion have both been described during or following COVID$19[44,45]$, which is thought to induce a systemic inflammatory response, endothelial dysfunction, and a hypercoagulative state, which predisposes patients to systemic thrombus formation [46].

Regarding post-vaccination thrombosis, rare cases of superior ophthalmic vein thrombosis and central retinal vein occlusion have been reported [47-50]. In this study were five cases of RVO (one CRVO and four BRVO), some of whom were affected by systemic comorbidities including DM or SAH. Moreover, a patient with no ocular or systemic risk factors reported unilateral NAION. So far, four cases of NAION associated with COVID-19 have been described in the literature [51-54].

Furthermore, three of our patients reported the activation or reactivation of a quiescent CNV secondary to myopia or uveitis

The literature reports a unilateral CSR 3 days after the injection of BNT162b2 mRNA COVID-19 vaccine occurred in a 33-year-old healthy Hispanic male without previous ocular history or pertinent medical history [55]. In our study, a case of acute-onset bilateral CSR in a male patient occurred 13 days after the second dose of BNT162b2 mRNA COVID19 vaccination. 
Most of the patients in our study (58.8\%) developed ocular complications after the second dose of the vaccine.

The main limitation of this study was its retrospective design and relatively low number of cases. Previous multiple reports have shown ocular complications following COVID19 vaccination, although a definitive association can be difficult to demonstrate. However, the close temporal association between vaccination and onset of uveitis or other ocular complications and the similarity to those reported in the literature are quite suggestive.

\section{Conclusions}

COVID-19 vaccination can be followed by herpetic keratitis reactivation in patients with previous herpetic keratitis or kerato-uveitis. The changes in the immune status, including lymphocyte exhaustion, may lead to herpes reactivation [25]. Therefore, prophylactic antiviral therapy with oral valacyclovir, at least for high-risk patients with several previous herpes uveitis episodes, may be considered.

COVID-19 vaccinations can also be followed by anterior scleritis; AU in patients with or without history of previous uveitis, and/or uveitis-related systemic disease; activation of Toxoplasma retinochoroiditis; VKH disease recurrences; pars planitis; retinal vasculitis; panuveitis in new-onset BD, MEWDS, and AMN; as well as RVO (CRVO or BRVO), NAION; activation of quiescent $\mathrm{CNV}$ secondary to myopia or uveitis; and CRS.

These complications could be related to the SARS-CoV-2 vaccines' capacity to induce autoimmune manifestations or thromboembolic events.

Additional epidemiologic and clinical studies and longer follow-up of this cohort are needed to confirm the link between the COVID-19 vaccine and the recurrence or de novo development of uveitis and other ocular complications.

Author Contributions: Conceptualization, L.C. and E.B.; methodology, L.C. and E.B.; data curation, V.M.; formal analysis, S.C., M.B., L.B. and A.Z.; investigation, L.D.S., F.G. and C.A.; writing-original draft preparation, E.B. and D.I.; writing — review and editing, E.B. and D.I.; visualization, E.B., S.C. and M.B.; supervision L.F., C.S. and L.C.; project administration, L.C. All authors have read and agreed to the published version of the manuscript.

Funding: This research received no external funding.

Institutional Review Board Statement: The study was conducted according to the guidelines of the Declaration of Helsinki, and approved by the local Ethics Committee (protocol n. 2021/0111389 Comitato Etico Provinciale di Reggio Emilia, Italy, date of approval 7 September 2021).

Informed Consent Statement: Informed consent was obtained from all subjects involved in the study. Data Availability Statement: Not applicable.

Conflicts of Interest: The authors declare no conflict of interest.

\section{References}

1. Gao, Q.; Bao, L.; Mao, H.; Wang, L.; Xu, K.; Yang, M.; Li, Y.; Zhu, L.; Wang, N.; Lv, Z.; et al. Development of an inactivated vaccine candidate for SARS-CoV-2. Science 2020, 369, 77-81. [CrossRef] [PubMed]

2. Xia, S.; Zhang, Y.; Wang, Y.; Wang, H.; Yang, Y.; Gao, G.F.; Tan, W.; Wu, G.; Xu, M.; Lou, Z.; et al. Safety and immunogenicity of an inactivated SARS-CoV-2 vaccine, BBIBP-CorV: A randomised, double-blind, placebo-controlled, phase 1/2 trial. Lancet Infect. Dis. 2021, 31, 39-51. [CrossRef]

3. Sadoff, J.; Gray, G.; Vandebosch, A.; Cárdenas, V.; Shukarev, G.; Grinsztejn, B.; Goepfert, P.A.; Truyers, C.; Fennema, H.; Spiessens, B.; et al. Safety and Efficacy of Single-Dose Ad26.COV2.S Vaccine against COVID-19. N. Engl. J. Med. 2021, 384, 2187-2201. [CrossRef] [PubMed]

4. Voysey, M.; Clemens, S.A.C.; Madhi, S.A.; Weckx, L.Y.; Folegatti, P.M.; Aley, P.K.; Angus, B.; Baillie, V.L.; Barnabas, S.L.; Bhorat, Q.E.; et al. Safety and efficacy of the ChAdOx1 nCoV-19 vaccine (AZD1222) against SARS-CoV-2: An interim analysis of four randomised controlled trials in Brazil, South Africa, and the UK. Lancet 2021, 397, 99-111. [CrossRef]

5. Polack, F.P.; Thomas, S.J.; Kitchin, N.; Absalon, J.; Gurtman, A.; Lockhart, S.; Perez, J.L.; Marc, G.P.; Moreira, E.D.; Zerbini, C.; et al. Safety and Efficacy of the BNT162b2 mRNA COVID-19 Vaccine. N. Engl. J. Med. 2020, 383, 2603-2615. [CrossRef]

6. Baden, L.R.; El Sahly, H.M.; Essink, B.; Kotloff, K.; Frey, S.; Novak, R.; Diemert, D.; Spector, S.A.; Rouphael, N.; Creech, C.B.; et al. Efficacy and Safety of the mRNA-1273 SARS-CoV-2 Vaccine. N. Engl. J. Med. 2021, 384, 403-416. [CrossRef] 
7. Heath, P.T.; Galiza, E.P.; Baxter, D.N.; Boffito, M.; Browne, D.; Burns, F.; Chadwick, D.R.; Clark, R.; Cosgrove, C.; Galloway, J.; et al. Safety and Efficacy of NVX-CoV2373 COVID-19 Vaccine. N. Engl. J. Med. 2021, 385, 1172-1183. [CrossRef]

8. McMahon, D.E.; Amerson, E.; Rosenbach, M.; Lipoff, J.B.; Moustafa, D.; Tyagi, A.; Desai, S.R.; French, L.E.; Lim, H.W.; Thiers, B.H.; et al. Cutaneous reactions reported after Moderna and Pfizer COVID-19 vaccination: A registry-based study of 414 cases. J. Am. Acad. Dermatol. 2021, 85, 46-55. [CrossRef]

9. Ng, X.L.; Betzler, B.K.; Testi, I.; Ho, S.L.; Tien, M.; Ngo, W.K.; Zierhut, M.; Chee, S.P.; Gupta, V.; Pavesio, C.E.; et al. Ocular Adverse Events After COVID-19 Vaccination. Ocul. Immunol. Inflamm. 2021, 1-9. [CrossRef]

10. The Standardization of Uveitis Nomenclature (SUN) Working Group. Standardization of uveitis nomenclature for reporting clinical data. Results of the first international workshop. Am. J. Ophthalmol. 2005, 140, 509-516. [CrossRef]

11. Benage, M.; Fraunfelder, F.W. Vaccine-Associated Uveitis. 2016. Mo Med. 2016, 113, 48-52.

12. Moorthy, R.S.; Moorthy, M.S.; Cunningham, E.T. Drug-induced uveitis. Curr. Opin. Ophthalmol. 2018, 29, 588-603. [CrossRef] [PubMed]

13. Cunningham, E.T.; Moorthy, R.S.; Fraunfelder, F.W.; Zierhut, M. Vaccine-Associated Uveitis. Ocul. Immunol. Inflamm. 2019, 27, 517-520. [CrossRef] [PubMed]

14. Cunningham, E.T.; Moorthy, R.S. Vaccine-Associated Posterior Uveitis. Retin. Cases Brief Rep. 2020, 40, 595-598.

15. Cheng, J.Y.; Margo, C.E. Ocular adverse events following vaccination: Overview and update. Surv. Ophthalmol. 2021, in press. [CrossRef] [PubMed]

16. Pichi, F.; Aljneibi, S.; Neri, P.; Hay, S.; Dackiw, C.; Ghazi, N.G. Association of Ocular Adverse Events With Inactivated COVID-19 Vaccination in Patients in Abu Dhabi. JAMA Ophthalmol. 2021, 139, 1131-1135. [CrossRef]

17. Rabinovitch, T.; Ben-Arie-Weintrob, Y.; Hareuveni-Blum, T.; Shaer, B.; Vishnevskia-Dai, V.; Shulman, S.; Newman, H.; Biadsy, M.; Masarwa, D.; Fischer, N.; et al. Uveitis following the BNT162b2 mRNA vaccination against SARS-CoV-2 infection. Retina 2021, 41, 2462-2471. [CrossRef]

18. Goyal, M.; Murthy, S.I.; Annum, S. Bilateral Multifocal Choroiditis following COVID-19 Vaccination. Ocul. Immunol. Inflamm. 2021, 29, 753-757. [CrossRef]

19. Mungmunpuntipantip, R.; Wiwanitkit, V. COVID-19 Vaccination and Bilateral Multifocal Choroiditis. Ocul. Immunol. Inflamm. 2021. [CrossRef] [PubMed]

20. Fernandez-Nieto, D.; Ortega-Quijano, D.; Suarez-Valle, A.; Burgos-Blasco, P.; Jimenez-Cauhe, J.; Fernandez-Guarino, M. Comment on: "To consider varicella-like exanthem associated with COVID-19, virus varicella zoster and virus herpes simplex must be ruled out. Characterization of herpetic lesions in hospitalized COVID-19 patients". J. Am. Acad. Dermatol. 2020, 83, e257-e259. [CrossRef] [PubMed]

21. Eid, E.; Abdullah, L.; Kurban, M.; Abbas, O. Herpes zoster emergence following mRNA COVID-19 vaccine. J. Med. Virol. 2021, 93, 5231-5232. [CrossRef]

22. Rodríguez-Jiménez, P.; Chicharro, P.; Cabrera, L.M.; Seguí, M.; Morales-Caballero, Á.; Llamas-Velasco, M.; Sánchez-Pérez, J. Varicella-zoster virus reactivation after SARS-CoV-2 BNT162b2 mRNA vaccination: Report of 5 cases. JAAD Case Reports 2021, 12, 58-59. [CrossRef] [PubMed]

23. Bostan, E.; Yalici-Armagan, B. Herpes zoster following inactivated COVID-19 vaccine: A coexistence or coincidence? J. Cosmet. Dermatol. 2021, 20, 1566-1567. [CrossRef] [PubMed]

24. Papasavvas, I.; De Courten, C.; Herbort, C.P., Jr. Varicella-zoster virus reactivation causing herpes zoster ophthalmicus ( HZO ) after SARS-CoV-2 vaccination-report of three cases. J. Ophthalmic Inflamm. Infect. 2021, 11, 28. [CrossRef]

25. Triantafyllidis, K.K.; Giannos, P.; Mian, I.T.; Kyrtsonis, G.; Kechagias, K.S. Varicella zoster virus reactivation following COVID-19 vaccination: A systematic review of case reports. Vaccines 2021, 9, 1013. [CrossRef]

26. Tartari, F.; Spadotto, A.; Zengarini, C.; Zanoni, R.; Guglielmo, A.; Adorno, A.; Valzania, C.; Pileri, A. Herpes zoster in COVID-19positive patients. Int. J. Dermatol. 2020, 59, 1028-1029. [CrossRef] [PubMed]

27. Herbort, C.P., Jr.; Papasavvas, I. Effect of SARS-CoV-2 mRNA vaccination on ocular herpes simplex and varicella-zoster virus reactivation : Should preventive antiviral treatment be given in known herpes patients. Ocul Immunol Inflamm. J. Ophthalmic Inflamm. Infect. 2021, 11, 33. [CrossRef] [PubMed]

28. Méndez Mangana, C.; Barraquer Kargacin, A.; Barraquer, R.I. Episcleritis as an ocular manifestation in a patient with COVID-19. Acta Ophthalmol. 2020, 98, e1056-e1057. [CrossRef]

29. Bostanci Ceran, B.; Ozates, S. Ocular manifestations of coronavirus disease. Graefe's Arch. Clin. Exp. Ophthalmol. 2020, 258, 1959-1963. [CrossRef]

30. Fernández Alcalde, C.; Granados Fernández, M.; Nieves Moreno, M.; Calvo Rey, C.; Falces Romero, I.; Noval Martín, S. COVID-19 ocular findings in children: A case series. World J. Pediatr. Springer Singapore 2021, 17, 329-334. [CrossRef]

31. Feizi, S.; Meshksar, A.; Naderi, A.; Esfandiari, H. Anterior Scleritis Manifesting After Coronavirus Disease 2019: A Report of Two Cases. Cornea 2021, 40, 1204-1206. [CrossRef] [PubMed]

32. Renisi, G.; Lombardi, A.; Stanzione, M.; Invernizzi, A.; Bandera, A.; Gori, A. Anterior uveitis onset after bnt162b2 vaccination: Is this just a coincidence? Int. J. Infect. Dis. 2021, 110, 95-97. [CrossRef]

33. Sprent, J.; King, C. COVID-19 vaccine side effects: The positives about feeling bad. Sci. Immunol. 2021, 6, 1-4. [CrossRef] [PubMed] 
34. Khan, I.A.; Ouellette, C.; Chen, K.; Moretto, M. Toxoplasma: Immunity and Pathogenesis. Curr. Clin. Microbiol. Reports 2019, 6, 44-50. [CrossRef] [PubMed]

35. Papasavvas, I.; Herbort, C.P. Reactivation of Vogt-Koyanagi-Harada disease under control for more than 6 years, following anti-SARS-CoV-2 vaccination. J. Ophthalmic Inflamm. Infect. 2021, 11, 21. [CrossRef]

36. Erdem, S.; Karahan, M.; Dursun, M.E.; Ava, S.; Hazar, L.; Katran, I.; Keklikci, U. Retinal Vasculitis Case Developing in the Early Period after COVID-19. Klin. Monbl. Augenheilkd. 2021. [CrossRef]

37. Insausti-García, A.; Reche-Sainz, J.A.; Ruiz-Arranz, C.; López Vázquez, Á.; Ferro-Osuna, M. Papillophlebitis in a COVID-19 patient: Inflammation and hypercoagulable state. Eur. J. Ophthalmol. 2020. [CrossRef]

38. Tagini, F.; Carrel, L.; Fallet, B.; Gachoud, D.; Ribi, C.; Monti, M. Behçet's-like adverse event or inaugural Behçet's disease after SARS-CoV-2 mRNA-1273 vaccination? Rheumatology 2021, 3-7. [CrossRef] [PubMed]

39. Virgo, J.; Mohamed, M. Paracentral acute middle maculopathy and acute macular neuroretinopathy following SARS-CoV-2 infection. Eye 2020, 34, 2352-2353. [CrossRef] [PubMed]

40. Bøhler, A.D.; Strøm, M.E.; Sandvig, K.U.; Moe, M.C.; Jørstad, Ø.K. Acute macular neuroretinopathy following COVID-19 vaccination. Eye 2021, 1-2. [CrossRef] [PubMed]

41. Book, B.A.J.; Schmidt, B.; Foerster, A.M.H. Bilateral Acute Macular Neuroretinopathy after Vaccination against SARS-CoV-19. JAMA Ophthalmol. 2021, 139, e212471. [CrossRef] [PubMed]

42. Mambretti, M.; Huemer, J.; Torregrossa, G.; Ullrich, M.; Findl, O.; Casalino, G. Acute Macular Neuroretinopathy following Coronavirus Disease 2019 Vaccination. Ocul. Immunol. Inflamm. 2021, 29, 730-733. [CrossRef] [PubMed]

43. Michel, T.; Stolowy, N.; Gascon, P.; Dupessey, F.; Comet, A.; Attia, R.; David, T.; Denis, D. Acute Macular Neuroretinopathy After COVID-19 Vaccine. J Ophthal Inflamm Infect. 2021, 1-9. [CrossRef]

44. Sheth, J.U.; Narayanan, R.; Goyal, J.; Goyal, V. Retinal vein occlusion in COVID-19: A novel entity. Indian J. Ophthalmol. 2020, 68, 2291-2293. [CrossRef]

45. Sen, M.; Honavar, S.G.; Sharma, N.; Sachdev, M.S. COVID-19 and eye: A review of ophthalmic manifestations of COVID-19. Indian J. Ophthalmol. 2021, 69, 488-509.

46. Haematology, T.L. COVID-19 coagulopathy: An evolving story. Lancet Haematol. 2020, 7, e425. [CrossRef]

47. Bayas, A.; Menacher, M.; Christ, M.; Behrens, L.; Rank, A.; Naumann, M. Bilateral superior ophthalmic vein thrombosis, ischaemic stroke, and immune thrombocytopenia after ChAdOx1 nCoV-19 vaccination. Lancet 2021, 397, e11. [CrossRef]

48. Panovska-Stavridis, I.; Pivkova-Veljanovska, A.; Trajkova, S.; Lazarevska, M.; Grozdanova, A.; Filipche, V. A rare case of superior ophthalmic vein thrombosis and thrombocytopenia following ChAdOx1 nCoV-19 vaccine against SARS-CoV-19. Mediterr. J. Hematol. Infect. Dis. 2021, 13, e2021048. [CrossRef]

49. Bialasiewicz, A.A.; Farah-Diab, M.S.; Mebarki, H.T. Central retinal vein occlusion occurring immediately after 2nd dose of mRNA SARS-CoV-2 vaccine. Int. Ophthalmol. 2021, 41, 3889-3892. [CrossRef]

50. Endo, B.; Bahamon, S.; Martínez-Pulgarín, D. Central retinal vein occlusion after mRNA SARS-CoV-2 vaccination: A case report. Indian J. Ophthalmol. 2021, 69, 2865-2866. [CrossRef]

51. Rho, J.; Dryden, S.C.; McGuffey, C.D.; Fowler, B.T.; Fleming, J. A Case of Non-Arteritic Anterior Ischemic Optic Neuropathy with COVID-19. Cureus 2020, 12, 10-12. [CrossRef] [PubMed]

52. Moschetta, L.; Fasolino, G.; Kuijpers, R.W. Non-arteritic anterior ischaemic optic neuropathy sequential to SARS-CoV-2 virus pneumonia: Preventable by endothelial protection? BMJ Case Rep. 2021, 14, 1-5. [CrossRef] [PubMed]

53. Clarke, K.M.; Riga, V.; Shirodkar, A.-L.; Meyer, J. Proning related bilateral anterior ischaemic optic neuropathy in a patient with COVID-19 related acute respiratory distress syndrome. BMC Ophthalmol. 2021, 21, 1-6. [CrossRef] [PubMed]

54. Girbardt, C.; Busch, C.; Al-Sheikh, M.; Gunzinger, J.M.; Invernizzi, A.; Xhepa, A.; Unterlauft, J.D.; Rehak, M. Retinal Vascular Events after mRNA and Adenoviral-Vectored COVID-19 Vaccines-A Case Series. Vaccines 2021, 9, 1349. [CrossRef] [PubMed]

55. Fowler, N.; Mendez Martinez, N.R.; Pallares, B.V.; Maldonado, R.S. Acute-onset central serous retinopathy after immunization with COVID-19 mRNA vaccine. Am. J. Ophthalmol. 2021, 23, 101136. [CrossRef] [PubMed] 